\title{
Abstract Word Definition in Patients with Amnestic Mild Cognitive Impairment
}

\author{
Soo Ryon Kim, ${ }^{1,2}$ SangYun Kim, ${ }^{3,4}$ Min Jae Baek, ${ }^{3,5}$ and HyangHee Kim ${ }^{1,6}$ \\ ${ }^{1}$ Graduate Program in Speech and Language Pathology, Yonsei University, Seoul 03722, Republic of Korea \\ ${ }^{2}$ Department of Speech and Hearing Therapy, Catholic University of Pusan, Busan 46252, Republic of Korea \\ ${ }^{3}$ Neurocognitive Behavior Center, Seoul National University Bundang Hospital, Seongnam 13620, Republic of Korea \\ ${ }^{4}$ Department of Neurology, Seoul National University College of Medicine, Seoul 03080, Republic of Korea \\ ${ }^{5}$ Translational Medicine, Seoul National University College of Medicine, Seoul 03080, Republic of Korea \\ ${ }^{6}$ Department and Research Institute of Rehabilitation Medicine, Yonsei University College of Medicine, Seoul 03722, Republic of Korea
}

Correspondence should be addressed to HyangHee Kim; h.kim@yonsei.ac.kr

Received 12 March 2015; Revised 21 July 2015; Accepted 26 July 2015

Academic Editor: Camillo Marra

Copyright (C) 2015 Soo Ryon Kim et al. This is an open access article distributed under the Creative Commons Attribution License, which permits unrestricted use, distribution, and reproduction in any medium, provided the original work is properly cited.

\begin{abstract}
The aims of this study were to investigate concrete and abstract word definition ability (1) between patients with amnestic mild cognitive impairment (aMCI) and normal adults and (2) between the aMCI subtypes (i.e., amnestic single-domain MCI and amnestic multidomain MCI; asMCI and amMCI) and normal controls. The 68 patients with aMCI (29 asMCI and 39 amMCI) and 93 age- and education-matched normal adults performed word definition tasks composed of five concrete (e.g., train) and five abstract nouns (e.g., jealousy). Task performances were analyzed on total score, number of core meanings, and number of supplementary meanings. The results were as follows. First, the aMCI patients scored significantly poorer than the normal controls in only abstract word definition. Second, both subtypes of aMCI performed worse than the controls in only abstract word definition. In conclusion, a definition task of abstract rather than concrete concepts may provide richer information to show semantic impairment of aMCI.
\end{abstract}

\section{Introduction}

Mild cognitive impairment (MCI) is characterized by multifarious changes in linguistic areas as well as in cognitive domains. The most prominent characteristic of MCI is impairment in semantic knowledge in contrast with relatively well preserved phonological, morphological, and syntactic knowledge [1]. Previous studies of MCI have mainly focused on semantic aspects, which were identified using diverse language tasks such as confrontation naming $[2,3]$, verbal fluency $[4,5]$, and discourse $[6,7]$. The confrontation naming tasks for patients with MCI have shown conflicting results, with one study demonstrating a difficulty with naming [1], while another showed no significant difference in naming ability compared with normal controls [2]. Likewise, the verbal fluency tasks had different outcomes due to the task types (e.g., categorical fluency, letter fluency) and the MCI experimental group characteristics $[4,5]$. Both the confrontation naming and verbal fluency tasks examine semantic retrieval ability at the word level [6] and are therefore limited for demonstrating subtle changes in cognition and language in patients with MCI.

Discourse tasks, which demand more comprehensive and natural linguistic capacity, have been implemented to compensate for this limitation. Patients with MCI were shown to produce inadequate information and a high proportion of empty utterances when presented with such tasks $[6,7]$. There are many types of discourse including narrative, procedural, expository, conversational, and descriptive. Different types and topics of discourse might generate diverse findings. In addition, discourse analysis is more time consuming than 
other language tasks and so may not be as well received for use in clinical settings.

To go elaborately into an examination of semantic characteristics of MCI, word definition tasks can be utilized. In word definition tasks, describing the meanings of words, the subjects' response is analyzed both in quantitative (i.e., numbers of semantic features produced for word definition) and qualitative (i.e., whether the semantic features are core or supplementary meanings) aspects [8]. The definition tasks, therefore, give a lot of information about the level of subjects' semantic processing. On the other hand, other semantic tasks require the subjects to generate the target words for their final response and consequently provide little information about the level of semantic processing achieved by them.

In spite of usefulness of word definition tasks for demonstrating subtle semantic degeneration, there are not many previous studies utilizing the tasks. Moreover, the researchers have most often carried on the tasks composed of only concrete words for various subject groups such as AD patients [9-11], normal elderly [12], children with language disorders [13], and normal children [14]. Concrete nouns are the names of tangible objects that one can experience or perceive with one's senses, which are the opposite of abstract nouns [15]. The conceptual knowledge for concrete and abstract nouns is stored in qualitatively different representational frameworks. Concrete concepts are supported in "categorical" frameworks, which have a close connection to super- and subordinate words in the same category. In contrast, abstract conceptualization involves integrated information in the "associative" framework. The associated features of abstract concepts have a horizontally spreading network [16]. Apart from the different representational frameworks, concrete and abstract concepts have different neural correlates. Based on neuroimaging studies, processing of high-level visual information of concrete words involves the left ventral temporal lobe whereas a high demand for semantic retrieval processing of abstract knowledge relies on the left inferior prefrontal lobe [17]. Finally, the development of definitions of concrete nouns takes place from preschool age, but that of abstract nouns is a gradual process from school age through adulthood, occurring over a relatively long period $[18,19]$. Consequently, these distinctive characteristics of concrete and abstract concepts could be differentially impaired through cognitive and linguistic declines with neurological diseases.

To the best of our knowledge, there is no study on semantic processing of both concrete and abstract concepts for MCI. Because MCI deteriorate into $\mathrm{AD}$ with a high proportion, we might be allowed to some insights on differential impairment in concrete and abstract semantics through the research for $\mathrm{AD}$. One study revealed that $\mathrm{AD}$ patients recalled more words with high imageability (i.e., concrete words) rather than those with low imageability (i.e., abstract words) in an immediate serial recall task. In addition, this deficit was accompanied with an abnormal proportion of phonological errors in the abstract word condition. In the synonym judgment task, AD patients also displayed selective impairment in abstract words comparing with concrete words [20]. Other researches have shown that $\mathrm{AD}$ patients have greater difficulties with abstract words than with concrete words in naming or semantic comprehension tasks [21-24]. Generally, the examinations of concrete and abstract knowledge for patients with AD have demonstrated relatively preserved concrete word processing in contrast with selective impairment in abstract concepts.

MCI is considered to be the intermediate stage from normal aging to dementia. Among the MCI subtypes, amnestic mild cognitive impairment (aMCI) is characterized as earlystage memory impairment and naming difficulty, and a large proportion of aMCI patients progress to Alzheimer's disease [25]. Recently, the determination of diagnosis subtypes such as amnestic single-domain MCI (asMCI) and amnestic multidomain MCI (amMCI) has become an important issue [26].

Thus, the purposes of this study were (1) to compare word definition ability (i.e., total score, number of core meanings, and number of supplementary meanings) between patients with aMCI and normal controls and (2) to identify any differences in word definition ability of aMCI patients with asMCI and amMCI subtypes compared to normal elderly.

\section{Methods}

2.1. Participants. This study included 68 patients with aMCI and 93 normal elderly patients. The aMCI patients were diagnosed by a neurologist according to the criteria proposed by Petersen et al.: (i) memory complaint usually corroborated by an informant; (ii) objective memory impairment for age; (iii) essentially preserved general cognitive function; (iv) largely intact functional activities; (v) being not demented [25]. All patients underwent neuropsychological tests using a standardized neuropsychological battery, called the Seoul Neuropsychological Screening Battery (SNSB) [27]. This battery covered tests for attention, language, praxis, the four symptoms of Gerstmann syndrome, visuoconstructive function, verbal and visual memory, and frontal/executive function.

On the basis of the profile of the neuropsychological tests, the aMCI patients were classified into two subtypes: 29 amnestic single-domain MCI (asMCI) patients with isolated memory dysfunction and 39 amnestic multidomain MCI (amMCI) patients with memory and other cognitive deficits.

Normal participants had no history of neurological or psychological disorders. They had adequate vision and hearing to perform the tasks. All participants scored within the normal range on the Korean version of the Mini-Mental State Examination (K-MMSE) [28] and had no symptoms of depression on the Geriatric Depression Scale Short FormKorean Version (GDSSF-K) [29].

The demographic characteristics of aMCI patients and healthy controls are shown in Table 1 . Independent $t$-tests revealed no significant differences between aMCI patients and normal controls with regard to age $(t=0.149, p>0.05)$, $\operatorname{sex}\left(\chi^{2}=0.221, p>0.05\right)$, or years of education $(t=-1.834$, $p>0.05)$. One-way ANOVA showed statistical differences among asMCI, amMCI, and normal controls with regard to years of education $(F=4.495, p<0.05)$ and MMSE scores $(F=5.068, p<0.01)$ but not age $(F=0.069, p>0.05)$ or sex $\left(\chi^{2}=0.299, p>0.05\right)$. The neuropsychological profile of the patients is also displayed in Table 2. 
TABle 1: Participant characteristics.

\begin{tabular}{|c|c|c|c|c|c|c|}
\hline Group & Subgroup & $N$ & Age (years) & $\operatorname{Sex}(M: F)$ & Education (years) & K-MMSE \\
\hline \multirow{3}{*}{ aMCI } & & 68 & $73.24 \pm 8.87$ & $29: 39$ & $12.63 \pm 4.26$ & $26.17 \pm 1.72$ \\
\hline & asMCI & 29 & $73.00 \pm 8.86$ & $13: 16$ & $12.48 \pm 4.14$ & $26.85 \pm 2.93$ \\
\hline & amMCI & 39 & $73.41 \pm 8.99$ & $16: 23$ & $13.62 \pm 4.10$ & $26.00 \pm 1.96$ \\
\hline Normal & & 93 & $72.80 \pm 8.46$ & $36: 57$ & $11.51 \pm 3.43$ & $27.33 \pm 2.02$ \\
\hline
\end{tabular}

Data are presented as mean \pm SD. aMCI: amnestic mild cognitive impairment. asMCI: amnestic single-domain mild cognitive impairment. amMCI: amnestic multidomain mild cognitive impairment. K-MMSE: Korean version of the Mini-Mental State Examination.

TABLE 2: Neuropsychological profile of patients.

\begin{tabular}{|c|c|c|c|}
\hline & $\begin{array}{c}\text { aMCI (total) } \\
(n=68)\end{array}$ & $\begin{array}{c}\text { asMCI } \\
(n=29)\end{array}$ & $\begin{array}{l}\text { amMCI } \\
(n=39)\end{array}$ \\
\hline \multicolumn{4}{|l|}{ Attention } \\
\hline Digit span forward test & $5.76 \pm 1.30$ & $5.78 \pm 1.48$ & $5.74 \pm 1.19$ \\
\hline Digit span backward test & $3.85 \pm 1.47$ & $3.89 \pm 1.22$ & $3.82 \pm 1.64$ \\
\hline \multicolumn{4}{|l|}{ Language function } \\
\hline K-BNT & $10.06 \pm 1.46$ & $10.78 \pm 2.21$ & $9.56 \pm 2.52$ \\
\hline \multicolumn{4}{|l|}{ Visuospatial function } \\
\hline RCFT copy & $30.50 \pm 4.12$ & $32.28 \pm 2.63$ & $29.27 \pm 4.53$ \\
\hline \multicolumn{4}{|l|}{ Memory function } \\
\hline SVLT immediate recall & $16.41 \pm 4.17$ & $17.74 \pm 3.93$ & $15.49 \pm 4.13$ \\
\hline SVLT delayed recall & $2.80 \pm 2.66$ & $3.48 \pm 2.86$ & $2.33 \pm 2.44$ \\
\hline SVLT recognition & $6.47 \pm 2.41$ & $6.70 \pm 2.69$ & $6.31 \pm 2.23$ \\
\hline RCFT immediate recall & $9.15 \pm 5.85$ & $10.20 \pm 5.77$ & $8.42 \pm 5.86$ \\
\hline RCFT delayed recall & $8.94 \pm 6.07$ & $10.04 \pm 5.92$ & $8.18 \pm 6.13$ \\
\hline RCFT recognition & $6.48 \pm 1.72$ & $6.72 \pm 1.80$ & $6.31 \pm 1.67$ \\
\hline \multicolumn{4}{|l|}{ Frontal function } \\
\hline Semantic COWAT (animal) & $13.20 \pm 4.13$ & $14.37 \pm 3.95$ & $12.38 \pm 4.10$ \\
\hline Phonemic COWAT & $7.11 \pm 3.36$ & $7.04 \pm 3.14$ & $7.15 \pm 3.54$ \\
\hline
\end{tabular}

Data are presented as mean \pm SD. aMCI: amnestic mild cognitive impairment. asMCI: amnestic single-domain mild cognitive impairment. amMCI: amnestic multidomain mild cognitive impairment. K-BNT: Korean version of Boston Naming Test. RCFT: Rey Complex Figure Test. SVLT: Seoul Verbal Learning Test (three free recall trials of 12 words and a 20-minute delayed recall trial and recognition test). COWAT: Controlled Oral Word Association Test.

We obtained informed consent from all participants, and the study was approved by the Institutional Review Boards of Seoul National University Bundang Hospital (IRB\#: B1402-240-106) for the patients with aMCI and of Severance Hospital (IRB\#: 1-2011-0061) for normal elderly controls.

2.2. Materials: Word Definition Task. We developed the word definition task to be composed of five concrete and five abstract nouns. The five concrete nouns (i.e., "watermelon," "rabbit," "train," "electric fan," and "pharmacy") were determined on the basis of semantic categories [30], definition categories [31], living and nonliving things [11], and imageability [9]. The representative semantic categories included vegetables, animals, transportations, electrical appliances, and places. Each word was associated with three or four definition categories (i.e., perceptual, functional, relational, and categorical). The relational and categorical definition categories were significantly less used by the patients with dementia compared to the normal controls, but the perceptual definition category showed no group differences [10].
Words prominent in only the perceptual definition category might not show age-related or neurological deterioration in word definition ability. In the next step, two words representing living things were chosen from the "animals" and "vegetables" categories. Two words representing man-made artifacts were also selected from the "transportations" and "electrical appliances" categories. The brain-damaged patients showed more severe impairment in the processing of living things than in the processing of nonliving things $[32,33]$. As living things have the characteristics of strong structural similarity and high visual complexity, the patients had difficulty processing such features [34]. Finally, a less imageable word was added to the concrete word list. Linguistic information is processed in the left hemisphere and visual-perceptual information in the right hemisphere. Therefore, words with high imageability are associated with both hemispheres. On the contrary, the left hemisphere is mainly activated when processing abstract concepts [35]. Due to the absence of standardized data on imageability, 20 normal adults (mean age: 27.03, mean education years: 15.13) rated the imageability of each word based on a 5-point 
TABLE 3: Examples of word definition task scoring.

\begin{tabular}{llll}
\hline Word & Participant definition & Core meanings & $\begin{array}{l}\text { Supplementary } \\
\text { meanings }\end{array}$ \\
\hline Watermelon (concrete noun) & $\begin{array}{l}\text { We usually eat it in summer. It is } \\
\text { cool. } \text { A fruit that we eat in a hot } \\
\text { summer because it is juicy. }\end{array}$ & $\begin{array}{l}\text { (1) Eat in summer } \\
\text { (2) A fruit }\end{array}$ & $\begin{array}{l}\text { (1) Cool } \\
\text { (2) Juicy }\end{array}$ \\
\hline Joke (abstract noun) & $\begin{array}{l}\text { A thing to make others laugh, } \\
\text { particularly among intimates. A } \\
\text { coarse joke is liable to be } \\
\text { misunderstood. }\end{array}$ & $\begin{array}{l}\text { (1) Make others } \\
\text { laugh } \\
\text { (2) Among } \\
\text { intimates }\end{array}$ & $\begin{array}{l}\text { Liable to be } \\
\text { misunderstood }\end{array}$ \\
\hline
\end{tabular}

Likert scale, through the questionnaire ("How imageable is this word?").

The five abstract words (i.e., "picnic," "jealousy," "music," "friendship," and "joke") were selected after considering semantic categories, clarity of semantic features, and abstractness. After investigating abstract words list employed in the previous studies $[19,36,37]$ and considering semantic categories of abstract concepts [38-40], we classified five semantic categories: emotion (e.g., happiness), traits (e.g., courage), social relation (e.g., friendship), mental state (e.g., consciousness), and action (e.g., performance). As abstract concepts do not directly correspond with entities in the physical world, as do concrete concepts, specific primary meanings are rarely fixed in abstract semantics [41]. As it is more difficult to define abstract nouns than concrete nouns, abstract concepts with clear semantic features were chosen for easier definition. Then, various levels of imageability were considered. The imageability of abstract nouns was investigated in the same way as with concrete nouns.

2.3. Procedures. After the participants filled out the demographic information and health state questionnaire, they completed the K-MMSE. For the word definition task, the participants were presented with concrete and abstract words in a random order on a computer screen. The examiner gave instructions to "Tell me what [sample word] is. Give me as much information about the word as possible." Thirty seconds were allowed for participants to define each word. Within 30 seconds, the instruction could be repeated when the participants produced no response or few utterances. The semantic features of each definition were classified into core meanings and supplementary meanings.

To score the subjects' responses to the word definition task, the criteria of core and supplementary meanings should be determined by priority. In this study, "analysis of range" was applied to provide objective and valid criteria for core and supplementary meanings. "Analysis of range" has been generally utilized in the vocabulary selection process, and it involves how many times a word was repeatedly selected in the previous studies and references. The high-range words are considered very important and the low-range words relatively less important [42]. In this study, analysis of range was applied by extracting the high-range semantic features from the subjects' utterance samples.
First, 100 normal elderly participants were made to perform a word definition task. Each semantic feature was extracted from a word definition. The range was determined by identifying how many times the semantic feature appeared in the 100 elderly participants' word definitions. A higher range among the subjects was taken to mean that the semantic feature more prominently occurs when describing a word meaning than those with a low range. Consequently, the semantic features produced by more than ten out of the 100 elderly participants for the word definition task were determined as core meanings. Each core meaning was assigned two points and each supplementary meaning was assigned one point. The points were summed to produce the total score of the word definition task. The examples of word definition task scoring appear in Table 3. All the responses were recorded using a Conic digital voice recorder S-10.

\subsection{Analysis}

2.4.1. Reliability Analysis. Interjudge reliability of coding for core and supplementary meanings was calculated by two researchers. One rater was the first author of this study, and the other rater was a doctoral graduate student in speech and language pathology. A total of 160 definitions (10\% of all responses) were randomly selected. Interjudge agreement was $93 \%$.

2.4.2. Data Analysis. The results were first analyzed to compare aMCI patients and normal controls. The total word definition, number of core meanings, and number of supplementary meanings for concrete noun scores were analyzed. The same analyses were performed for abstract nouns. An independent $t$-test was performed to confirm the differences between two groups whose age, sex, and years of education were matched.

Second, asMCI, amMCI, and normal elderly patients were compared to investigate any differences in total score, number of core meanings, and number of supplementary meanings. We performed one-way analysis of covariance (ANCOVA) after adjusting for years of education and MMSE score. $p$ values $<0.05$ were considered statistically significant. Statistical analyses were carried out using SPSS 19.0 for Windows. 
TABLE 4: Word definition task performance for aMCI patients and normal controls.

\begin{tabular}{lcccc}
\hline & & aMCI patients & Normal controls & $p$ value \\
\hline \multirow{3}{*}{ Concrete word } & Total score & $27.61 \pm 7.20$ & $30.34 \pm 8.91$ & .72 \\
& No. of core meanings & $11.55 \pm 3.15$ & $12.52 \pm 3.57$ & .125 \\
& No. of supplementary meanings & $4.50 \pm 2.65$ & $5.31 \pm 3.26$ & .145 \\
\hline \multirow{3}{*}{ Abstract word } & Total score & $19.45 \pm 5.87$ & $22.23 \pm 6.85$ & $.020^{*}$ \\
& No. of core meanings & $8.46 \pm 2.80$ & $.15 \pm 2.97$ & .204 \\
& No. of supplementary meanings & $2.52 \pm 2.02$ & $3.94 \pm 2.31$ & $.001^{* *}$ \\
\hline
\end{tabular}

Data are presented as mean \pm SD. aMCI: amnestic mild cognitive impairment. No.: number.

${ }^{*} p<.05,{ }^{* *} p<.01$.

TABLE 5: Word definition task performance for asMCI, amMCI, and normal controls.

\begin{tabular}{|c|c|c|c|c|c|}
\hline & & asMCI patients & amMCI patients & Normal controls & $p$ value \\
\hline \multirow{3}{*}{ Concrete word } & Total score & $29.37 \pm 6.27$ & $29.28 \pm 8.61$ & $28.61 \pm 8.92$ & .948 \\
\hline & No. of core meanings & $12.63 \pm 2.65$ & $11.92 \pm 3.55$ & $11.56 \pm 3.61$ & .383 \\
\hline & No. of supplementary meanings & $4.11 \pm 2.50$ & $5.44 \pm 3.08$ & $5.50 \pm 3.59$ & .103 \\
\hline \multirow{3}{*}{ Abstract word } & Total score & $21.17 \pm 8.61$ & $20.85 \pm 6.85$ & $20.91 \pm 7.21$ & .975 \\
\hline & No. of core meanings & $8.85 \pm 2.84$ & $9.37 \pm 3.16$ & $8.42 \pm 3.27$ & .466 \\
\hline & No. of supplementary meanings & $3.49 \pm 2.40$ & $2.11 \pm 1.57$ & $4.07 \pm 2.73$ & $.001^{* *}$ \\
\hline
\end{tabular}

Data are presented as mean \pm SD. asMCI: amnestic single-domain mild cognitive impairment. amMCI: amnestic multidomain mild cognitive impairment. No.: number.

${ }^{* *} p<.01$.

\section{Results}

3.1. Comparison of Word Definition Abilities between Patients with Amnestic Mild Cognitive Impairment and Normal Controls. For the performances on concrete word definition task between aMCI patients and normal controls (Table 4), the $t$ test showed no statistical differences in total score $(t=1.818$, $p>0.05)$, number of core meanings $(t=1.545, p>0.05)$, or number of supplementary meanings $(t=1.466, p>0.05)$. However, for abstract nouns between two groups, the $t$-test showed statistical differences in total score $(t=2.353, p<$ $0.05)$ and number of supplementary meanings $(t=3.526$, $p<0.01)$ but not in number of core meanings $(t=1.276$, $p>0.05)$.

3.2. Comparison of Word Definition Abilities among Patients with Amnestic Single-Domain MCI, Amnestic Multidomain MCI, and Normal Controls. After adjusting for years of education and MMSE score, total score, number of core meanings, and number of supplementary meanings were compared among the three groups. The analysis of ANCOVA revealed no differences with regard to concrete nouns. However, the ANCOVA showed a significant difference in number of supplementary meanings of abstract nouns $(p<0.01)$ but not in total score or number of core meanings. Post hoc analyses indicated that all three groups were different with regard to number of supplementary meanings $(p<0.001)$ as shown in Table 5.

\section{Discussion}

Contextual information can be specified to process traits of "abstractness," because abstract semantics is learned via use in sentences and association with other concepts [33]. A specific primary meaning is rarely fixed in the processing of abstract semantics because it does not correspond directly with entities in the physical world, as do concrete concepts [41]. In the present study, aMCI patients demonstrated a significant deficit in abstract noun definition tasks compared to normal elderly individuals. Additionally, both aMCI subtypes (amnestic single-domain MCI and amnestic multidomain MCI) had difficulty producing sufficient semantic features of abstract words.

There are at least three hypotheses to account for the semantic impairment in abstract concepts identified in aMCI patients. First, the impairment is based on the "concreteness effect." Concrete concepts are more easily processed than abstract concepts. The Paivio's dual-code perspective [35] explains that concrete concepts rely on both language and sensorimotor information (i.e., verbal and nonverbal code), whereas abstract concepts are supported only by language (i.e., verbal code). In addition to the dual-code theory, concrete words have more related contextual information than abstract words [43], and they are described by a greater number of semantic features [44]. All of these explanations support the idea that concrete concepts take advantage of semantic processing than do abstract concepts. This explains the compatible performance on concrete noun definition tasks for aMCI individuals compared to the normal controls.

Second, there are different representational frameworks between abstract and concrete concepts. It has recently been proposed that abstract concepts are supported by an "associative" neural network and that abstract concepts are semantically connected with other associated knowledge in a horizontally arranged network. In contrast, concrete concepts 
are organized by category and have semantic features in super- and subordinate relations $[17,45]$. According to the "spreading activation model" [46], the distances between concepts are differentiated by categorical relation, typicality, and the associated degrees of other concepts. A semantically long distance prevents rapid and strong activation through word processing. This semantically spreading integration occurs in the prefrontal regions [47] that are vulnerable to MCI. Therefore, patients with MCI have difficulty with semantically divergent activations, only processing primary meanings that have a close distance to a target word. As a result, only a partial specification of word meanings can be achieved, producing fewer supplementary meanings of an abstract word.

The third hypothesis to account for selective impairment in abstract word definition is that concrete and abstract concepts are different in preponderant semantic information. Affective information plays a greater role for abstract concepts, while sensory-motor information is more prominent for concrete concepts. Some researchers reported that abstract words have an advantage on processing semantic knowledge due to their greater affective associations [48, 49]. However, strong affective valence of abstract concepts might hinder proper description of word meanings and allow individuals to focus on their personal feelings and experiences related to the target word. For example, while defining the word of "music," the word evoked abnormally strong positive or negative emotion. The patients could not generate appropriate semantic features but started to talk about their favorite music and singer and even ended in singing or crying.

Although aMCI patients exhibited abstract concept deficits, they demonstrated comparable performance levels in concrete noun definition tasks compared with the normal elderly group. These findings did not replicate those of the previous study that implemented a concrete word definition task for patients with MCI [50]. In Lim et al's study, patients with MCI revealed significantly lower scores than the control in a 22-concrete word definition task. However, the differences in findings of the current study and Lim et al.s may be attributable to the following three reasons: first one could be due to methodological differences such as the numbers of subjects (e.g., 68 versus 8) that were employed in the studies. Second reason could be ascribed to MCI subtype specification. The previous study did not classify MCI subtypes. However, in this current study, we divided the subtypes of MCI because each subtype could be heterogeneously distinctive in nature [25]. Specifically, the patients that were classified as aMCI have demonstrated greater progression to Alzheimer's disease and are considered to be a significant pathological group. The third reason for the difference was that they employed different scoring methods. Whereas the previous study used a 3-point equal-interval scoring method (e.g., 0,1 , and 2 points), we adopted total scores (sum of core and supplementary meanings) which may be more multidimensional and, therefore, more sensitive to subtle pathological group differences [51].

Intactness in core and superordinate knowledge in word concepts observed in aMCI has not been found in $\mathrm{AD}$ patients. The previous studies of $\mathrm{AD}$ patients demonstrated a qualitatively weaker definition ability and fewer primary features of target words compared with the normal $[9,10]$. In this study, distinction was made between aMCI and AD patients which may serve as a linguistic-behavioral marker for differentiating the two.

The results of the study are noteworthy because it is the first study to utilize abstract nouns in word definition task to examine semantic knowledge impairment in patients with aMCI. Abstract words (e.g., "sensible," "confident") in registration and recall tasks have been included in the recently developed Mini-Mental State Examination, 2nd edition (MMSE-2) [52]. The MMSE-2 was developed to provide finer discrimination as a reliable cognitive screening measure because the previous version of the MMSE has been known to be less sensitive for detecting patients with MCI and in the early stages of dementia [53].

In conclusion, a definition task of abstract rather than concrete concepts may provide richer information to show semantic impairment of aMCI. Semantic deficits of aMCI may often go unnoticed due to their mild nature, which in turn requires highly sensitive linguistic tasks for detecting semantic deficits. Future study is needed to confirm the process of semantic knowledge deteriorated from aMCI to various stages of $\mathrm{AD}$.

\section{Disclosure}

Part of this paper was presented in the doctoral dissertation submitted to the Graduate Program in Speech and Language Pathology, the Graduate School of Yonsei University, Seoul, Republic of Korea, in December 2014.

\section{Conflict of Interests}

The authors declare no conflict of interests.

\section{Acknowledgment}

This work was supported by a National Research Foundation (the Ministry of Education, Science and Technology) grant funded by the Korean Government (NRF-2011-32A-B00203) given to Professor HyangHee Kim.

\section{References}

[1] K. A. Bayles and A. W. Kaszniak, Communication and Cognition in Normal Aging and Dementia, College Hill Press, Boston, Mass, USA, 1987.

[2] A. Duong, V. Whitehead, K. Hanratty, and H. Chertkow, "The nature of lexico-semantic processing deficits in mild cognitive impairment," Neuropsychologia, vol. 44, no. 10, pp. 1928-1935, 2006.

[3] I. Daum, G. Riesch, G. Sartori, and N. Birbaumer, "Semantic memory impairment in Alzheimer's disease," Journal of Clinical and Experimental Neuropsychology, vol. 18, no. 5, pp. 648-665, 1996. 
[4] A. Weakley, M. Schmitter-Edgecombe, and J. Anderson, "Analysis of verbal fluency ability in amnestic and non-amnestic mild cognitive impairment," Archives of Clinical Neuropsychology, vol. 28, no. 7, pp. 721-731, 2013.

[5] K. E. Nutter-Upham, A. J. Saykin, L. A. Rabin et al., "Verbal fluency performance in amnestic MCI and older adults with cognitive complaints," Archives of Clinical Neuropsychology, vol. 23, no. 3, pp. 229-241, 2008.

[6] H. Choi, "Performances in a picture description task in Japanese patients with Alzheimer's disease and with mild cognitive impairment," Communication Sciences and Disorders, vol. 14, no. 3, pp. 326-337, 2009.

[7] V. Fleming and J. Harris, "Complex discourse production in mild cognitive impairment: detecting subtle changes," Aphasiology, vol. 22, no. 7-8, pp. 729-740, 2008.

[8] M. Bierwisch and F. Kiefer, "Remarks on definitions in natural language," in Studies in Syntax and Semantics, F. Kiefer, Ed., Riedel, Dordrecht, The Netherlands, 1970.

[9] A. J. Astell and T. A. Harley, "Accessing semantic knowledge in dementia: evidence from a word definition task," Brain and Language, vol. 82, no. 3, pp. 312-326, 2002.

[10] J. R. Hodges, K. Patterson, N. Graham, and K. Dawson, "Naming and knowing in dementia of Alzheimer's type," Brain and Language, vol. 54, no. 2, pp. 302-325, 1996.

[11] S. C. Abeysinghe, K. A. Bayles, and M. W. Trosset, "Semantic memory deterioration in Alzheimer's subjects: evidence from word association, definition, and associate ranking tasks," Journal of Speech and Hearing Research, vol. 33, no. 3, pp. 574-582, 1990.

[12] S. Kim and H. Choi, "Characteristics of verbal definitions in normal elderly Koreans," Communication Sciences and Disorders, vol. 17, no. 1, pp. 107-117, 2012.

[13] S. B. Lee and H. R. Lee, "Definitional category analysis of ambiguous words in Korean children with specific language impairment," Journal of Speech and Hearing Disorders, vol. 16, no. 4, pp. 1-18, 2007.

[14] K. A. Park and Y. T. Kim, "A comparative study of verbal definitions of concrete nouns between normal children and languagedelayed children," Communication Sciences and Disorders, vol. 5, no. 2, pp. 20-37, 2005.

[15] NAKL, Korean Standard Unabridged Dictionary, National Academy of Korean Language, 1999.

[16] S. K. Scott, “The neural representation of concrete nouns: what's right and what's left?” Trends in Cognitive Sciences, vol. 8, no. 4, pp. 151-153, 2004.

[17] S. J. Crutch, "Qualitatively different semantic representations for abstract and concrete words: further evidence from the semantic reading errors of deep dyslexic patients," Neurocase, vol. 12, no. 2, pp. 91-97, 2006.

[18] M. A. Nippold, S. L. Hegel, M. M. Sohlberg, and I. E. Schwarz, "Defining abstract entities: development in pre-adolescents, adolescents, and young adults," Journal of Speech, Language, and Hearing Research, vol. 42, no. 2, pp. 473-481, 1999.

[19] B. McGhee-Bidlack, "The development of noun definitions: a metalinguistic analysis," Journal of Child Language, vol. 18, no. 2, pp. 417-434, 1991.

[20] F. Peters, S. Majerus, J. De Baerdemaeker, E. Salmon, and F. Collette, "Impaired semantic knowledge underlies the reduced verbal short-term storage capacity in Alzheimer's disease," Neuropsychologia, vol. 47, no. 14, pp. 3067-3073, 2009.
[21] E. Albanese, “The 'hidden' semantic category dissociation in mild-moderate Alzheimer's disease patients," Neuropsychologia, vol. 45, no. 4, pp. 639-643, 2007.

[22] J. F. Kroll and J. S. Merves, "Lexical access for concrete and abstract words," Journal of Experimental Psychology: Learning, Memory, and Cognition, vol. 12, no. 1, pp. 92-107, 1986.

[23] M. Rissenberg and M. Glanzer, "Free recall and word finding ability in normal aging and senile dementia of the Alzheimer's type: the effect of item concreteness," Journals of Gerontology, vol. 42, no. 3, pp. 318-322, 1987.

[24] H.-A. Yi, P. Moore, and M. Grossman, "Reversal of the concreteness effect for verbs in patients with semantic dementia," Neuropsychology, vol. 21, no. 1, pp. 9-19, 2007.

[25] R. C. Petersen, "Mild cognitive impairment as a diagnostic entity," Journal of Internal Medicine, vol. 256, no. 3, pp. 183-194, 2004.

[26] A. Busse, A. Hensel, U. Gühne, M. C. Angermeyer, and S. G. Riedel-Heller, "Mild cognitive impairment: long-term course of four clinical subtypes," Neurology, vol. 67, no. 12, pp. 2176-2185, 2006.

[27] Y. W. Kang and D. L. Na, Seoul Neuropsychological Screening Battery (SNSB), Human Brain Research \& Consulting, Incheon, Republic of Korea, 2003.

[28] Y. W. Kang, "A normative study of the Korean-Mini Mental State Examination (K-MMSE) in the elderly," Korean Journal of Psychology, vol. 25, no. 2, pp. 1-12, 2006.

[29] B. S. Kee, "A preliminary study for the standardization of geriatric depression scale short form-Korea version," Journal of Korean Neuropsychiatric Association, vol. 35, no. 2, pp. 298-307, 1996.

[30] A. Raymer and L. J. Gonzalez-Rothi, "Clinical diagnosis and treatment of naming disorders," in The Handbook of Adult Language Disorders: Integrating Cognitive, Neuropsychology, Neurology, and Rehabilitation, A. E. Hillis, Ed., pp. 163-184, Taylor \& Francis, New York, NY, USA, 2002.

[31] K. A. Bayles, "Effects of working memory deficits on the communicative functioning of Alzheimer's dementia patients," Journal of Communication Disorders, vol. 36, no. 3, pp. 209-219, 2003.

[32] R. Barbarotto, E. Capitani, H. Spinnler, and C. Trivelli, "Slowly progressive semantic impairment with category specificity," Neurocase, vol. 1, no. 2, pp. 107-119, 1995.

[33] S. D. Breedin, E. M. Saffran, and H. B. Coslett, "Reversal of the concreteness effect in a patient with semantic dementia," Cognitive Neuropsychology, vol. 11, no. 6, pp. 617-660, 1994.

[34] R. J. S. Wise, D. Howard, C. J. Mummery et al., "Noun imageability and the temporal lobes," Neuropsychologia, vol. 38, no. 7, pp. 985-994, 2000.

[35] A. Paivio, "Dual coding theory: retrospect and current status," Canadian Journal of Psychology, vol. 45, no. 3, pp. 255-287, 1991.

[36] J. F. Marques and L. D. Nunes, “The contributions of language and experience to the representation of abstract and concrete words: different weights but similar organizations," Memory and Cognition, vol. 40, no. 8, pp. 1266-1275, 2012.

[37] U. Noppeney and C. J. Price, "Retrieval of abstract semantics," NeuroImage, vol. 22, no. 1, pp. 164-170, 2004.

[38] M. Ghio, M. M. S. Vaghi, and M. Tettamanti, "Fine-grained semantic categorization across the abstract and concrete domains," PLoS ONE, vol. 8, no. 6, Article ID e67090, 2013.

[39] E. Catricalà, P. A. Della Rosa, V. Plebani, G. Vigliocco, and S. F. Cappa, "Abstract and concrete categories? Evidences from 
neurodegenerative diseases," Neuropsychologia, vol. 64, pp. 271281, 2014.

[40] P. A. Della Rosa, E. Catricalà, S. de Battisti, D. Vinson, G. Vigliocco, and S. F. Cappa, "How to assess abstract conceptual knowledge: construction, standardization and validation of a new battery of semantic memory tests," Functional Neurology, vol. 29, no. 1, pp. 47-55, 2014.

[41] E. M. Saffran, M. F. Schwartz, and M. C. Linebarger, "Context availability and lexical decisions for abstract and concrete words," Journal of Memory and Language, vol. 27, no. 5, pp. 449$520,1998$.

[42] H. Lee, "A study on loanword selection for Korean language learners," Poetics and Linguistics, vol. 27, pp. 151-180, 2014.

[43] P. J. Schwanenflugel and E. J. Shoben, "Differential context effects in the comprehension of abstract and concrete verbal materials," Journal of Experimental Psychology: Learning, Memory, and Cognition, vol. 9, no. 1, pp. 82-102, 1983.

[44] D. C. Plaut and T. Shallice, "Deep dyslexia: a case study of connectionist neuropsychology," Cognitive Neuropsychology, vol. 10, no. 5, pp. 377-500, 1993.

[45] S. J. Crutch and E. K. Warrington, "Abstract and concrete concepts have structurally different representational frameworks," Brain, vol. 128, no. 3, pp. 615-627, 2005.

[46] A. M. Collins and E. F. Loftus, "A spreading-activation theory of semantic processing," Psychological Review, vol. 82, no. 6, pp. 407-428, 1975.

[47] A. Dietrich and R. Kanso, "A review of EEG, ERP, and neuroimaging studies of creativity and insight," Psychological Bulletin, vol. 136, no. 5, pp. 822-848, 2010.

[48] S.-T. Kousta, G. Vigliocco, D. P. Vinson, M. Andrews, and E. Del Campo, "The representation of abstract words: why emotion matters," Journal of Experimental Psychology: General, vol. 140, no. 1, pp. 14-34, 2011.

[49] G. Vigliocco, S.-T. Kousta, P. A. Della Rosa et al., "The neural representation of abstract words: the role of emotion," Cerebral Cortex, vol. 24, no. 7, pp. 1767-1777, 2014.

[50] S. Lim, M. Kwon, H. S. Sim, S. Kim, and J. Y. Lee, "Word definition ability in patients with Alzheimer's disease," Dementia and Neurocognitive Disorders, vol. 13, no. 1, pp. 7-15, 2014.

[51] B. E. Porch, Porch Index of Communicative Ability, Consulting Psychological Press, Palo Alto, Calif, USA, 3rd edition, 1981.

[52] M. F. Folstein, S. E. Folstein, T. White, and M. A. Messer, MiniMental State Examination, Psychological Assessment Resources, Lutz, Fla, USA, 2nd edition, 2010.

[53] D. Galasko, L. A. Hansen, R. Katzman et al., "Clinical-neuropathological correlations in Alzheimer's disease and related dementias," Archives of Neurology, vol. 51, no. 9, pp. 888-895, 1994. 


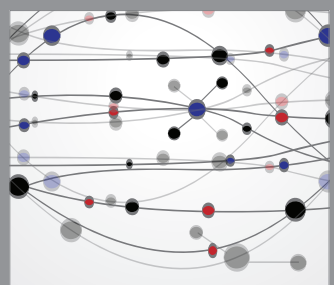

The Scientific World Journal
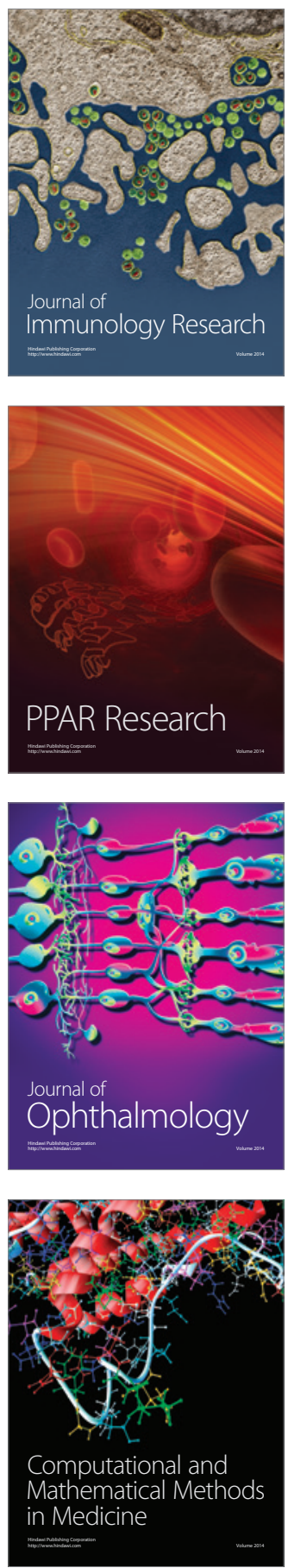

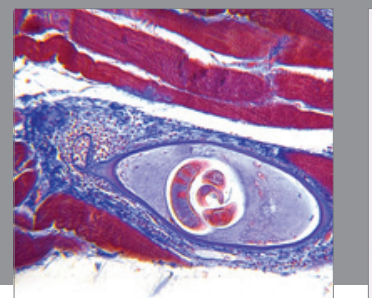

Gastroenterology

Research and Practice
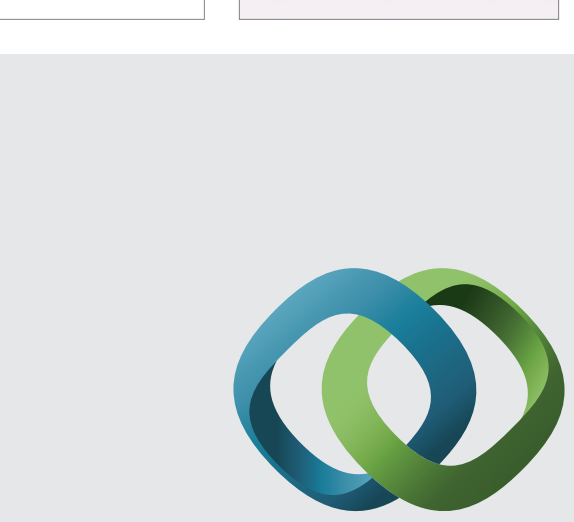

\section{Hindawi}

Submit your manuscripts at

http://www.hindawi.com
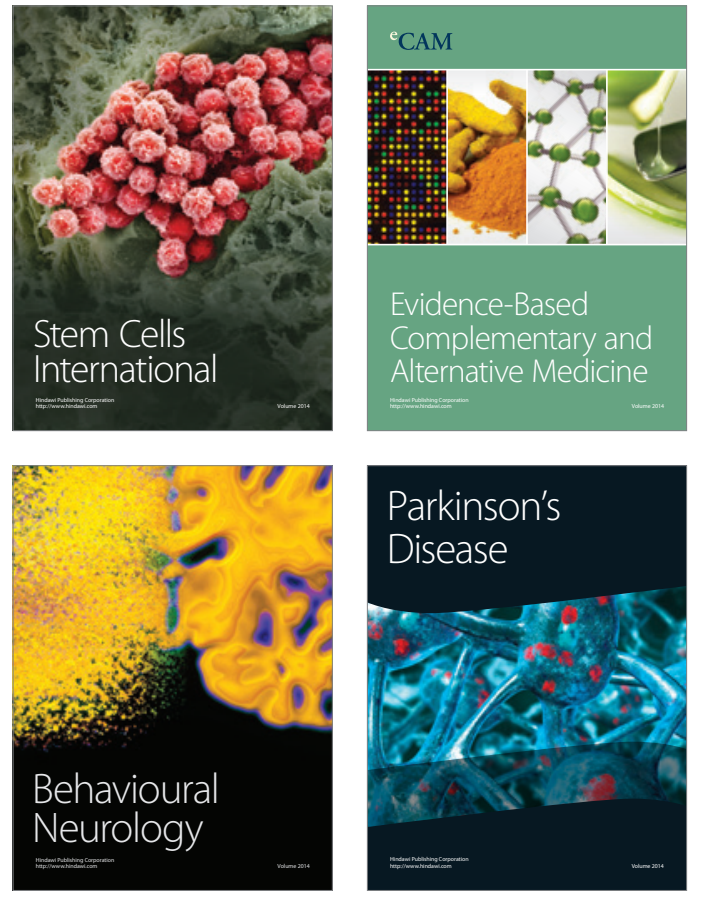
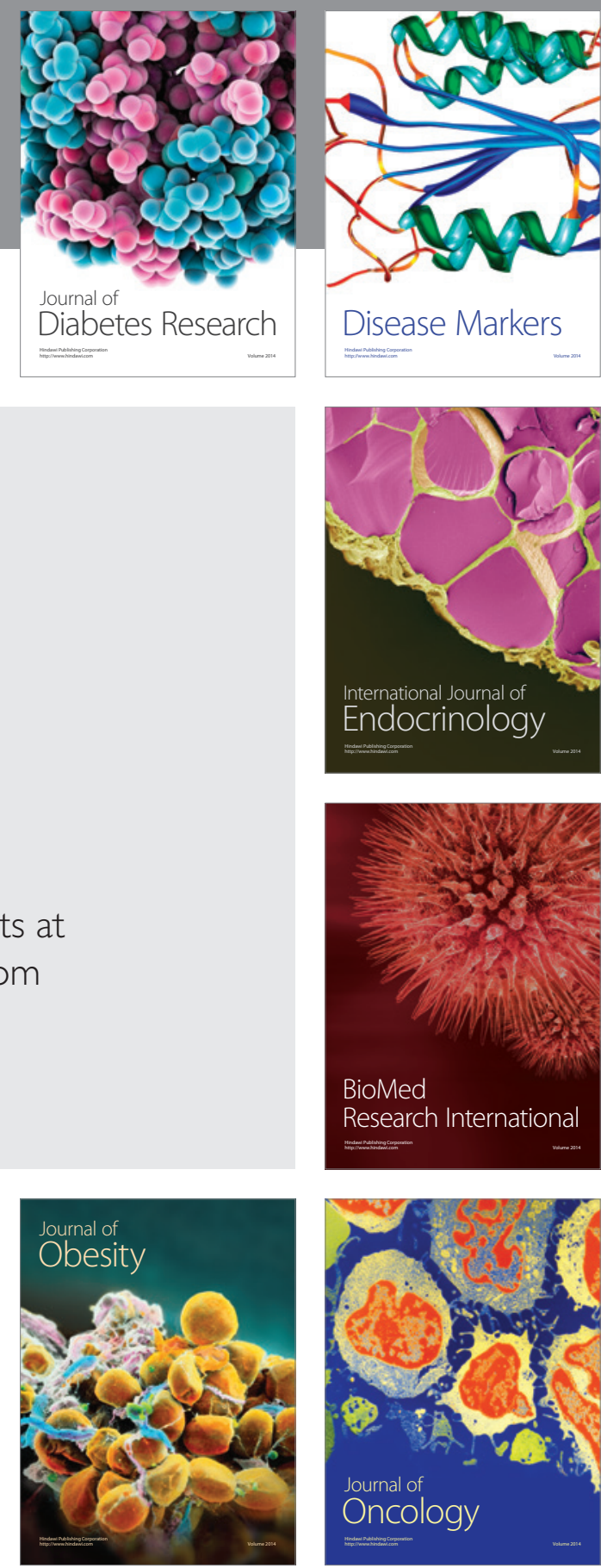

Disease Markers
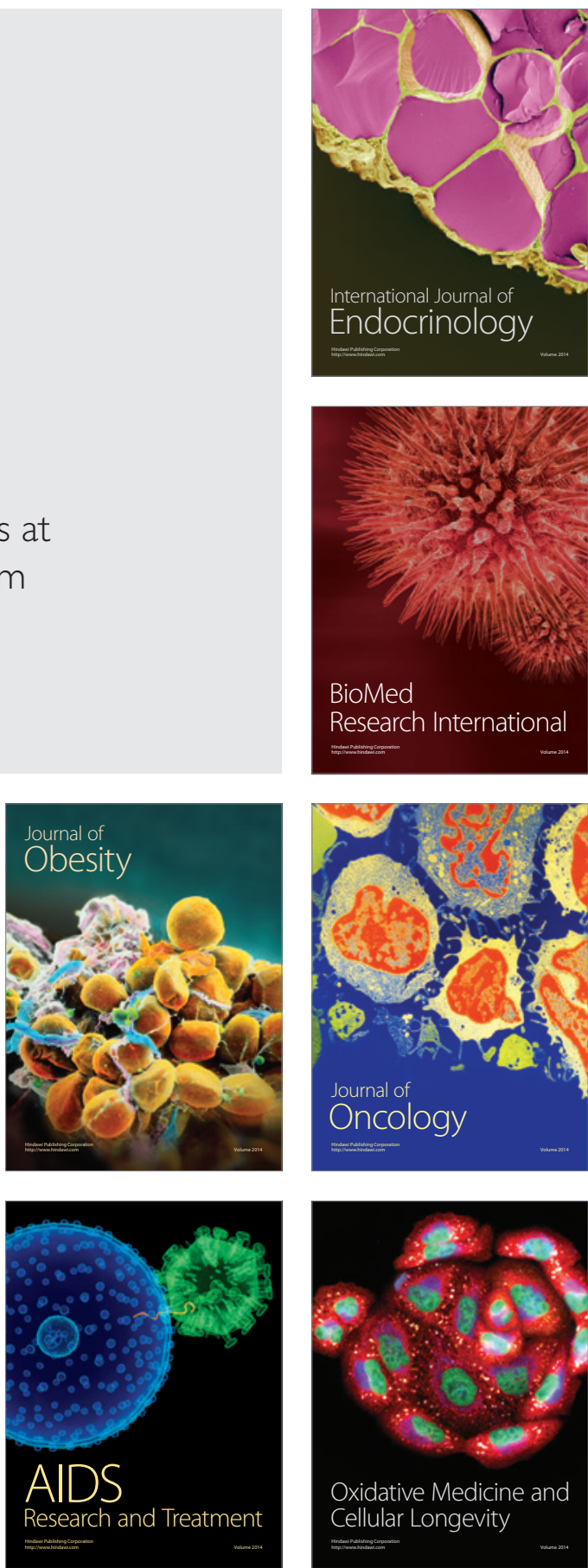